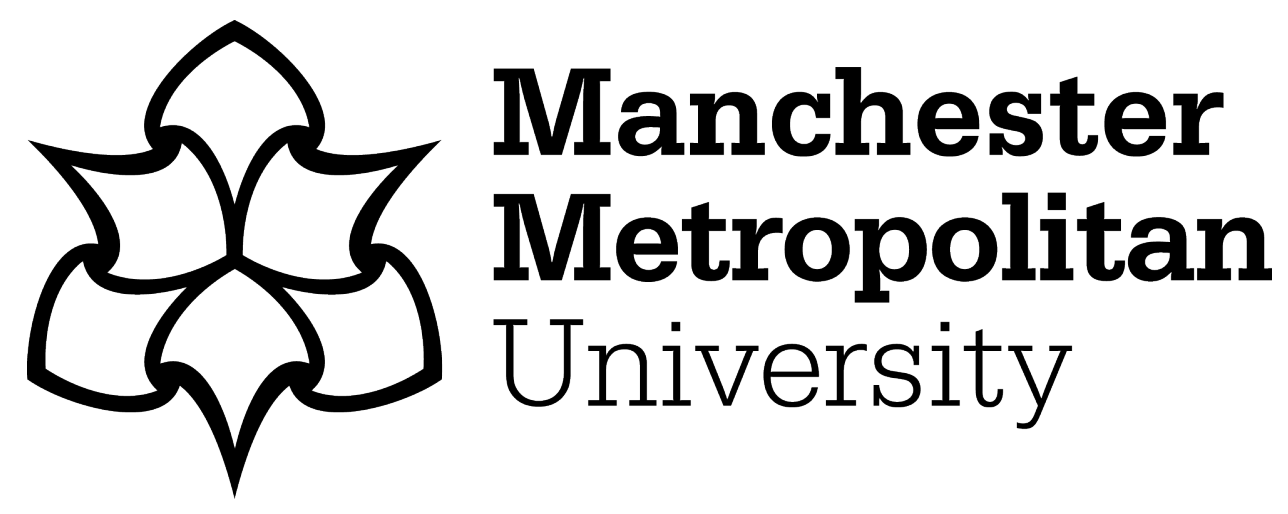

Soltanpur, C, Paravi, R, Ghamari, M and Adebisi, B ORCID logoORCID: https://orcid.org/0000-0001-9071-9120 (2019) Nonlinear MMSE Equalizer for Impulsive Noise Mitigation in OFDM-Based Communications. IEEE Signal Processing Letters, 26 (7). pp. 1016-1020. ISSN 1070-9908

Downloaded from: https://e-space.mmu.ac.uk/624370/

Version: Accepted Version

Publisher: IEEE

DOI: https://doi.org/10.1109/LSP.2019.2917018

Please cite the published version 


\title{
Nonlinear MMSE Equalizer for Impulsive Noise Mitigation in OFDM-Based Communications
}

\author{
C. Soltanpur, R. Paravi, M. Ghamari, and B. Adebisi
}

\begin{abstract}
Destructive effects of impulsive noise has been broadly observed not only in wireless communication systems but also in power line communications (PLCs). Impulsive noise is a common impediment in orthogonal frequency division multiplexing (OFDM)-based communication systems for industry applications. This non-Gaussian noise degrades the performance of conventional equalizers and hence elicit a modified version of the equalizer that fits the non-Gaussian description of the noise. This paper proposes a nonlinear minimum mean square error (MMSE) equalizer for OFDM systems where the characteristics of the added noise to the system is known. The soft values were obtained based on the derivation of the equalizer for a memoryless channel impaired with impulsive noise. Obtaining such values are required for the implementation of turbo-equalization scheme. The validity of such an equalizer is tested through simulations and the result of simulations show that the nonlinear equalizer is successful in combating the effect of an impulsive noise. Thus, the turbo-coded OFDM system shows a significant boost at low signal-to-noise ratios.
\end{abstract}

\section{INTRODUCTION}

Impulsive noise is a common impediment in achieving error-free transmission in orthogonal frequency division multiplexing (OFDM) systems that are broadly used in various communications standards such as wireless [1], power line [2], in-vehicle [3], digital subscriber line [4], and underwater communications [5]. Communication systems based on multi-carrier transmission schemes such as OFDM systems are shown to be more resilient to non-Gaussian impulsive interferences in comparison to single-carrier systems due to random distribution of their noise energy over multiple subcarriers [6],[7]. However, under specific circumstances, when the energy of impulsive noise exceeds a certain threshold level, impulsive noise can be very destructive and greatly influence the performance of OFDM systems. Examples of such scenarios are given in [8] and [9]. So far, several heuristic methods such as blanking [10], [7] and clipping [11], [12] have been proposed in the literature to combat the effect of non-Gaussian impulsive noise [13]. However, as of now, the aforementioned methods have not been successful in providing an insight into the calculation of the soft values at the output of equalizer - an important statistic for decoding the transmitted signal.

This paper studies an optimum nonlinear minimum mean squared error (MMSE) equalizer [14], based on Masreliez work in [15], [16], that can potentially mitigate the effects of impulsive noise in OFDM-based communication systems. The optimality condition is derived under weak conditions widely used in MMSE equalizer [17]. We used this nonlinear equalizer to assess the transmission reliability of a communication system. To that end, we consider a Middleton class-A noise model [18] and employ an error control coding technique to have a better understanding of the limits of operation in harsh environments for our proposed receiver. Main contribution of this work is the efficient calculation of soft values in presence of an impulsive noise. We further investigate the impact of the turbo-equalization [19] scheme on the overall performance of the system. The equalizer presented in this work is limited to a memory-less channel. The generalization to a channel with inter-symbol interference (ISI) is explained in [14].

\section{COMMUNiCATIONS SYSTEM}

The notations used in this paper are as follows: Bold uppercase and bold lowercase letters denote matrices and vectors, respectively. Conjugate transpose is denoted by $(.)^{H}$. The notation $\|$.$\| represents Euclidean norm for vectors and |$.$| is$ the absolute value of a complex number. Circularly symmetric, complex Gaussian distribution with mean $\mu$ and variance $\sigma^{2}$ is denoted by $\mathcal{C N}\left(\mu, \sigma^{2}\right)$; I is the identity matrix and $\operatorname{diag}[$. represents a diagonal matrix whose diagonal elements are given.

\section{A. System Model}

A basic block diagram of the system considered in this paper is shown in Fig. 1. We assume a block of modulated symbols of size $\mathrm{N}$, denoted as $\mathbf{x}=\left[x_{0}, \ldots, x_{N-1}\right]^{T}$, where $x_{k}$ chosen from alphabet set $\mathcal{X}$, is passed through inverse Fourier transform block to produce $\tilde{\mathbf{x}}=\left[\tilde{x}_{0}, \ldots, \tilde{x}_{N-1}\right]^{T}$. The cyclic prefix of appropriated size is added before transmission and removed on the other receiver side. The time domain signal can be expressed as

$$
\tilde{y}_{k}=\sum_{k=0}^{K} h_{k} \tilde{x}_{(n-k) \equiv N}+\tilde{n}_{k},
$$

where $h_{k}, k=0, \ldots, K$, represents the channel impulse response and $n_{k}$ is the noise. The indexes of x's are chosen congruent to $0, \ldots, \mathrm{N}-1$ modulo $\mathrm{N}$. To retrieve the original signal a fast Fourier transform (FFT) is performed on the received block. Alternatively, the output of channel in (1) can be expressed in vector form as

$$
\tilde{\mathbf{y}}=\mathbf{H} \tilde{\mathbf{x}}+\tilde{\mathbf{n}},
$$

where $\mathbf{H}$ is the cyclic channel response matrix [20]. The diagonalized matrix of channel response matrix using discrete Fourier transform (DFT) is represented by $\overline{\mathbf{H}}$.

On the receiver side, as shown in Fig. 1, the received signal in time domain is shared between an inverse FFT module and a module to compute the nonlinear filter taps. Once the coefficients of the filter is passed through an inverse FFT module and set, the symbols in frequency domain are filtered 


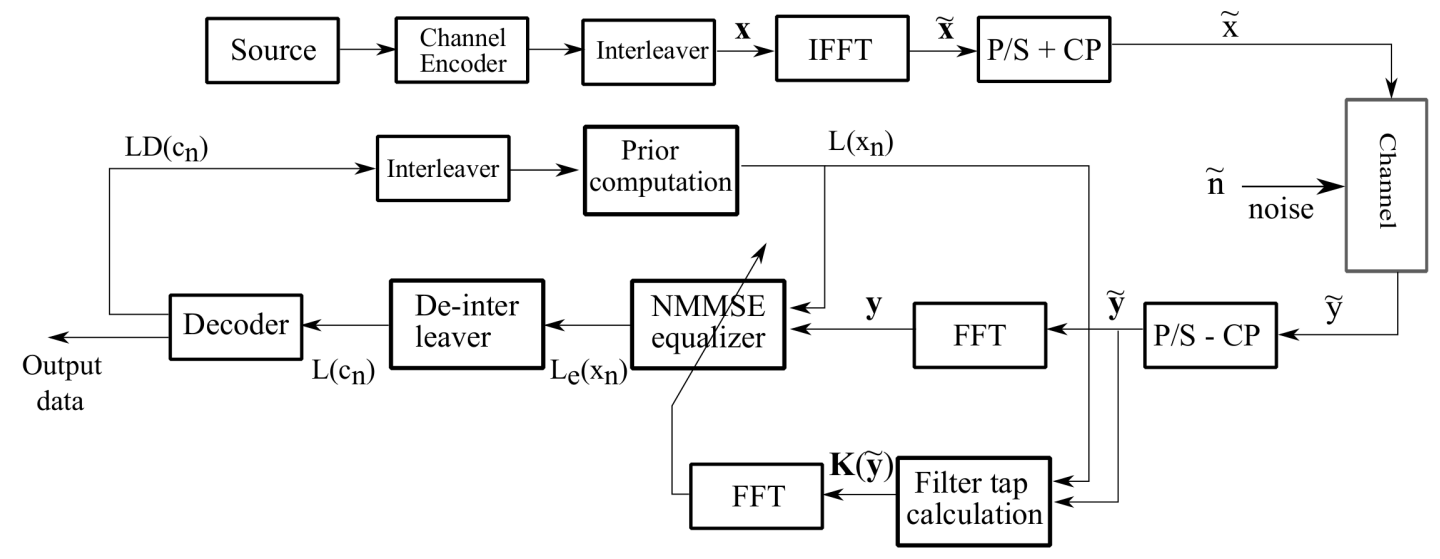

Fig. 1. Block diagram of nonlinear MMSE filter for OFDM system equipped with turbo-equalization.

out to compute the extrinsic information. The soft values at the output of decoder closes the turbo-equalization loop. Note that computed a priori information from the decoder are used to both update the equalizer coefficients and the equalizer output.

\section{B. Impulsive Noise}

The probability density function of the complex Class-A noise [21], [18], designated as $p_{\Omega}$, is given by

$$
p_{\Omega}(\tilde{n})=\sum_{m=0}^{\infty} \frac{a_{m}}{\pi \sigma_{m}^{2}} \exp \left(-\frac{|\tilde{n}|^{2}}{\sigma_{m}^{2}}\right),
$$

where $a_{m}$ and $\sigma_{m}^{2}$ are given respectively as

$$
a_{m}=\frac{e^{-A} A^{m}}{m !}, \quad \sigma_{m}^{2}=\sigma^{2}\left(\frac{m / A+\Gamma}{1+\Gamma}\right),
$$

where $\sigma^{2}$ is the variance of the total noise, $m$ denotes the channel state $(0,1,2, \ldots)$ and $\sigma_{m}^{2}$ represents the noise variance for channel state $m$ [22]. The parameter $A$ is referred to as the impulsive index and $\Gamma$ is the Gaussian-to-impulse noise power ratio. There are three parameters specifying the statistical characteristics of this model, namely: $A, \Gamma$ and $\sigma^{2}$. When $A$ is increased, the noise will be similar to a Gaussian noise; reduced values of $A$ means high noise pulses. The distribution stated in (3) can be seen as a Gaussian mixture [23]. To make the computation tractable, we truncate the number of Gaussian terms to the first $K$ terms [24]. This approximation has shown to be sufficient to represent the Class-A noise distribution [24]. Truncating to the first $K$ terms, we rewrite the approximate Class-A noise distribution as

$$
p_{\Omega}\left(\tilde{n}_{k}\right)=\sum_{k=0}^{K} a_{k}^{\prime} \mathcal{C N}\left(0, \sigma_{k}^{2}\right)
$$

where $a_{k}^{\prime}=a_{k} / \sum_{k=0}^{K} a_{k}$.

\section{EQUALIZER DESIGN FOR NON-GAUSSIAN NoISE}

Using the orthogonality principle, we can write the estimator of transmitted signal as conditional expectation of transmitted signal given the output of the channel

$$
\hat{\mathbf{x}}=\mathbb{E}_{\mathbf{x}}[\mathbf{x} \mid \tilde{\mathcal{Y}}=\tilde{\mathbf{y}}]
$$

The derivation of estimator is given in [14]. The estimator for the transmitted information $\mathbf{x}$ can be stated as

$$
\hat{\mathbf{x}}=\mathbf{x}^{p r i}-\mathbf{U}^{H} \boldsymbol{\Sigma}_{\tilde{\mathbf{x}}} \mathbf{H}^{H} \frac{\partial}{\partial \tilde{\mathbf{y}}^{H}} \ln p_{Y}(\tilde{\mathbf{y}}) .
$$

where $\mathbf{U}$ is the unitary DFT matrix, and superscript "pri" means a priori. The Masreliez equalizer in (7) is derived under assumption that elements of $\tilde{\mathbf{x}}$ are the weighted sum of random variables and, based on the central limit theorem [25], can be approximated by Gaussian distribution [17]. The derived estimator is the best estimator according to minimum mean squared error (MMSE), however, it is nonlinear.

The covariance of error of the estimate in (7) is stated as follows

$$
\boldsymbol{\Sigma}_{\hat{\mathbf{x}}}=\mathbb{E}_{\mathbf{x}}\left[(\hat{\mathbf{x}}-\mathbf{x})(\hat{\mathbf{x}}-\mathbf{x})^{H} \mid \tilde{\mathcal{Y}}=\tilde{\mathbf{y}}\right]
$$

The derivation of the error covariance matrix is omitted for brevity and is given in [15]. For non-Gaussian noise covariance of error is a nonlinear function of the probability of received signal is

$$
\boldsymbol{\Sigma}_{\hat{\mathbf{x}}}=\mathbf{U}^{H}\left[\boldsymbol{\Sigma}_{\tilde{\mathbf{x}}}^{p r i}+\boldsymbol{\Sigma}_{\tilde{\mathbf{x}}}^{p r i} \mathbf{H}^{H} \mathbf{G}(\tilde{\mathbf{y}}) \mathbf{H} \boldsymbol{\Sigma}_{\tilde{\mathbf{x}}}^{p r i}\right] \mathbf{U},
$$

where matrix $\mathbf{G}(\tilde{\mathbf{y}})$ is defined by its elements as $[\mathbf{G}(\tilde{\mathbf{y}})]_{i, j}=$ $\frac{\partial^{2}}{\partial \tilde{y}_{j} \partial \tilde{y}_{i}^{*}} \ln p(\tilde{\mathbf{y}})$. In the absence of prior information we set $\mathbf{x}^{p r i}=\mathbf{0}$ and $\boldsymbol{\Sigma}_{\tilde{\mathbf{x}}}=\sigma_{\tilde{x}}^{2} \mathbf{I}$. The equalizer presented here reduces to the known MMSE equalizer for the Gaussian noise [26].

For impulsive noise, we define diagonal matrix $\mathbf{K}(\tilde{\mathbf{y}})$ by its elements $[\mathbf{K}(\tilde{\mathbf{y}})]_{k, k}=\frac{\partial}{\partial \tilde{y}_{k}^{*}}\left(\ln p_{Y}(\tilde{\mathbf{y}})\right) /\left(\tilde{y}_{k}-h_{k} \tilde{x}_{k}^{p r i}\right)$, so we can further write (7) and (9) as

$$
\begin{array}{r}
\hat{\mathbf{x}}=\mathbf{x}^{p r i}-\boldsymbol{\Sigma}_{\mathbf{x}} \overline{\mathbf{H}}^{H} \mathbf{U}^{H} \mathbf{K}(\tilde{\mathbf{y}}) \mathbf{U}\left(\mathbf{y}-\overline{\mathbf{H}} \mathbf{x}^{p r i}\right), \\
\boldsymbol{\Sigma}_{\hat{\mathbf{x}}}=\boldsymbol{\Sigma}_{\mathbf{x}}{ }^{p r i}+\boldsymbol{\Sigma}_{\mathbf{x}}{ }^{p r i} \overline{\mathbf{H}}^{H} \mathbf{U}^{H} \mathbf{G}(\tilde{\mathbf{y}}) \mathbf{U} \overline{\mathbf{H}} \boldsymbol{\Sigma}_{\mathbf{x}}{ }^{p r i}
\end{array}
$$

For simplicity let us assume the channel has no memory. For treatment of a channel with ISI we refer the reader to [14]. The assumption that channel has no ISI allows us to simplify the probability of received signal in (7). The integral and product are interchangeable in derivation of $p_{Y}$ as

$$
p_{Y}(\tilde{\mathbf{y}})=\prod_{k=1}^{N} \int p_{\Omega}\left(\tilde{y}_{k}-h_{k} \tilde{x}_{k}\right) p\left(\tilde{x}_{k}\right) d \tilde{x}_{k},
$$


where we assumed that the elements of vector $\tilde{\mathbf{x}}_{k}$ are independent. For impulsive noise distribution given in (3) we have

$$
\begin{gathered}
p_{Y}\left(\tilde{y}_{k}\right)=\sum_{m=0}^{\infty} \int \frac{a_{m}}{\pi \sigma_{m}^{2}} \exp \left(\frac{-\left|\tilde{y}_{k}-h_{k} \tilde{x}_{k}\right|^{2}}{\sigma_{m}^{2}}\right) p\left(\tilde{x}_{k}\right) d \tilde{x}_{k} \\
=\sum_{m=0}^{\infty} \frac{a_{m} / \pi}{\sigma_{m}^{2}+\sigma_{\tilde{x}_{k}}^{2}\left|h_{k}\right|^{2}} \exp \left(\frac{-\left|\tilde{y}_{k}-h_{k} \tilde{x}_{k}^{p r i}\right|^{2}}{\sigma_{m}^{2}+\sigma_{\tilde{x}_{k}}^{2}\left|h_{k}\right|^{2}}\right)
\end{gathered}
$$

Last equality resulted from substituting $\mathcal{C N}\left(x_{k}^{p r i}, \sigma_{x_{k}}^{2}\right)$ for $p(\tilde{x})$. For brevity let the two auxiliary variables $\dot{b}$ and $\ddot{b}$ be defined by

$$
\begin{aligned}
& \dot{b}\left(\tilde{y}_{k}\right)=\sum_{m=0}^{\infty} \frac{a_{m} / \pi}{\left(\sigma_{m}^{2}+\sigma_{\tilde{x}_{k}}^{2}\left|h_{k}\right|^{2}\right)^{2}} \exp \left(\frac{-\left|\tilde{y}_{k}-h_{k} \tilde{x}_{k}^{p r i}\right|^{2}}{\sigma_{m}^{2}+\sigma_{\tilde{x}_{k}}^{2}\left|h_{k}\right|^{2}}\right), \\
& \ddot{b}\left(\tilde{y}_{k}\right)=\sum_{m=0}^{\infty} \frac{a_{m} / \pi}{\left(\sigma_{m}^{2}+\sigma_{\tilde{x}_{k}}^{2}\left|h_{k}\right|^{2}\right)^{3}} \exp \left(\frac{-\left|\tilde{y}_{k}-h_{k} \tilde{x}_{k}^{p r i}\right|^{2}}{\sigma_{m}^{2}+\sigma_{\tilde{x}_{k}}^{2}\left|h_{k}\right|^{2}}\right) .
\end{aligned}
$$

Yielding the derivative in Masreliez equalizer as

$$
\frac{\partial}{\partial \tilde{y}_{k}^{*}} \ln p_{Y}\left(\tilde{y}_{k}\right)=-\frac{\dot{b}\left(\tilde{y}_{k}\right)}{p_{Y}\left(\tilde{y}_{k}\right)}\left(\tilde{y}_{k}-h_{k} \tilde{x}_{k}^{p r i}\right) .
$$

The proposed filter at limit will reduce to blanking filter which is introduced in [27]. To calculate the error covariance matrix we need to calculate the diagonal elements of matrix $\mathbf{G}$ as follows

$$
\begin{aligned}
\frac{\partial^{2}}{\partial \tilde{y}_{k} \partial \tilde{y}_{k}^{*}} \ln p_{Y}\left(\tilde{y}_{k}\right)=\frac{-1}{p_{Y}^{2}\left(\tilde{y}_{k}\right)} & \frac{\partial}{\partial \tilde{y}_{k}^{*}} p_{Y}\left(\tilde{y}_{k}\right) \\
& +\frac{1}{p_{Y}(\tilde{y})} \frac{\partial^{2}}{\partial \tilde{y}_{k} \partial \tilde{y}_{k}^{*}} p_{Y}\left(\tilde{y}_{k}\right) .
\end{aligned}
$$

If we plug in the impulse noise distribution given in (3) the second derivative reduces to

$$
\begin{aligned}
\frac{\partial^{2}}{\partial \tilde{y}_{k} \partial \tilde{y}_{k}^{*}} \ln p_{Y}\left(\tilde{y}_{k}\right) & =\frac{-\dot{b}\left(\tilde{y}_{k}\right)}{p_{Y}\left(\tilde{y}_{k}\right)}+ \\
\mid \tilde{y}_{k} & -\left.h_{k} \tilde{x}_{k}^{p r i}\right|^{2}\left[\frac{\ddot{b}\left(\tilde{y}_{k}\right)}{p_{Y}\left(\tilde{y}_{k}\right)}-\frac{\dot{b}^{2}\left(\tilde{y}_{k}\right)}{p_{Y}^{2}\left(\tilde{y}_{k}\right)}\right] .
\end{aligned}
$$

\section{Updating Soft VAlues}

In this section, we discuss the soft values for the equalizer derived in Section III. At the output of equalizer the loglikelihood ratio (LLR) values are used as input to the decoder. For Gaussian noise, LLR values are calculated in [28]. To extend these result to the non-Gaussian noise we start with calculating the extrinsic information $L_{e}\left(x_{n}\right)$ which is passed on as a priori LLR $L\left(c_{n}\right)$ for the decoder. For turbo equalization scheme, as explained in [29], the equalizer takes the $a$ priori LLR from the decoder, depicted as $L(x)$ in Fig. 1. The extrinsic information is used to update the decoder's a priori LLRs.

We opt to use the Gaussian mixture approximation to express the mean and the variance of received symbols. Recall that the impulsive noise can be approximated by Gaussian mixture distribution as explained in Section II.B. Using the Gaussian mixture distribution, we can write the simplified mean and the variance of received signal based on the following

$$
\mathbf{G}(\tilde{\mathbf{y}})=\mathbf{K}(\tilde{\mathbf{y}})=\operatorname{diag}\left[-\sum_{m=0}^{K} \frac{p\left(M=m \mid \tilde{y}_{n}\right)}{\sigma_{m}^{2}+\sigma_{\tilde{x}_{n}}^{2}|h|^{2}}\right] .
$$

In (19), using Bayes' rule we can further estimate current state and characteristics of the noise based on the received signal as follows

$$
p\left(M=m \mid \tilde{y}_{n}\right)=\frac{p_{Y}\left(\tilde{y}_{n} \mid M=m\right) p(M=m)}{\sum_{k=0}^{K} p_{Y}\left(\tilde{y}_{n} \mid m\right) p(M=m)},
$$

where we set $p(M=m)=a_{m}^{\prime}$.

In order to find the extrinsic information at the output of detector, we start with the estimate of $x$ from (7). We use the conditional distribution $p\left(\hat{x}_{n} \mid x_{n}=\alpha\right)$ to derive the extrinsic information $L_{e}\left(x_{n}\right)$. It is commonly assumed in the literature [30] that the conditional distribution $p\left(\hat{x}_{n} \mid x_{n}=\alpha\right)$ has a Gaussian shape with mean and variance given by $\mu_{n}=$ $\mathbb{E}_{Y}\left[\hat{x}_{n} \mid x_{n}=\alpha\right]$ and $v_{n}^{2}=\mathbb{E}_{Y}\left[\hat{\sigma}_{x_{n}}^{2} \mid x_{n}=\alpha\right]$, respectively. We start by deriving the mean and variance in the time domain. In matrix form we have

$$
\hat{x}_{n}=x_{n}^{p r i}-\sigma_{x_{n}}^{2} \bar{h}_{n}^{*} \mathbf{u}_{n}^{H} \mathbf{K}(\tilde{\mathbf{y}}) \mathbf{U}\left(\mathbf{y}-\overline{\mathbf{H}} \mathbf{x}^{p r i}\right) .
$$

We can see from (21) that $\hat{\mathbf{x}}$ is given by the weighted sum of received samples. We fix the $n$-th transmitted symbol as $\alpha$, then the mean and the variance of $\tilde{\mathbf{x}}$ are designated by $\tilde{\mu}$ and $\tilde{\mathbf{V}}$ for transmitted symbols through the channel. The mean of $\hat{\tilde{\mathbf{x}}}$ with respect to received signal is

$$
\tilde{\mu}=\tilde{\mathbf{x}}^{p r i}+\boldsymbol{\Sigma}_{\tilde{\mathbf{x}}} \mathbf{H}^{H} \mathbf{K H U}\left[\begin{array}{c}
\mathbf{0} \\
\left(\alpha-x_{n}^{p r i}\right) \\
\mathbf{0}
\end{array}\right],
$$

where $\mathbf{K}=\operatorname{diag}\left[\sum_{m=0}^{K} a_{m}^{\prime}\left(\sigma_{m}^{2}+\sigma_{\tilde{x}_{n}}^{2}|h|^{2}\right)^{-1}\right]$. Note that $\mathbf{K}$ does not depend on $\tilde{\mathbf{y}}$ anymore. Covariance matrix of our estimate is equivalent to Fisher information matrix [31] as follows

$$
\tilde{\mathbf{V}}=\boldsymbol{\Sigma}_{\tilde{\mathbf{x}}} \mathbf{H}^{H} \operatorname{var}[\mathbf{K}(\tilde{\mathbf{y}}) \tilde{\mathbf{y}} \mid \alpha] \mathbf{H} \boldsymbol{\Sigma}_{\tilde{\mathbf{x}}}
$$

Unfortunately, the covariance matrix does not yield a closed form. For practical applications to avoid the computationally prohibitive numerical calculation we would use the following approximation: for a large number of symbols the observed noise in frequency domain, by central limit theorem, can be described as $\sigma^{2} \mathbf{I}$, where $\sigma$ is defined in (4). With this assumption the variance in (23) reduces to the Gaussian case as given in [28].

Finally, we can write the mean and variance of $\hat{x}_{n}$ as

$$
\begin{gathered}
\mu_{n}=x_{n}^{p r i}+\sigma_{x_{n}}^{2}\left|\bar{h}_{n}\right|^{2} \mathbf{u}_{n}^{H} \mathbf{K} \mathbf{U}\left[\begin{array}{c}
\mathbf{0} \\
\left(\alpha_{n}-x_{n}^{p r i}\right) \\
\mathbf{0}
\end{array}\right], \\
v_{n}^{2}=\frac{\sigma_{x_{n}}^{4}\left|\bar{h}_{n}\right|^{2}}{\left(\sigma^{2}+\sigma_{x_{n}}^{2}\left|\bar{h}_{n}\right|^{2}\right)}-\frac{\sigma_{x_{n}}^{6}\left|\bar{h}_{n}\right|^{4}}{\left(\sigma^{2}+\sigma_{x_{n}}^{2}\left|\bar{h}_{n}\right|^{2}\right)^{2}} .
\end{gathered}
$$

Once we have the distribution of $p\left(\hat{x}_{n} \mid x_{n}=\alpha\right)$ the extrinsic information can be written as stated in [19]. 


\section{Simulations}

In this section, the performance of nonlinear equalizer developed in this paper is examined against different impulsive noise parameters. The noise is generated based on three term approximation of model given in (3). An OFDM system with $N=128$ subcarriers is used for simulations with binary phase shift keying (BPSK) modulated symbols. The channel is assumed to be known, Rayleigh distributed, and memoryless. Turbo code with code rate of 0.3125 is employed as forward error correction coding [32]. This is done by recursive systemic encoder for the code generator with octal notation $\mathbf{g}=[3,17]$ as described in [33].

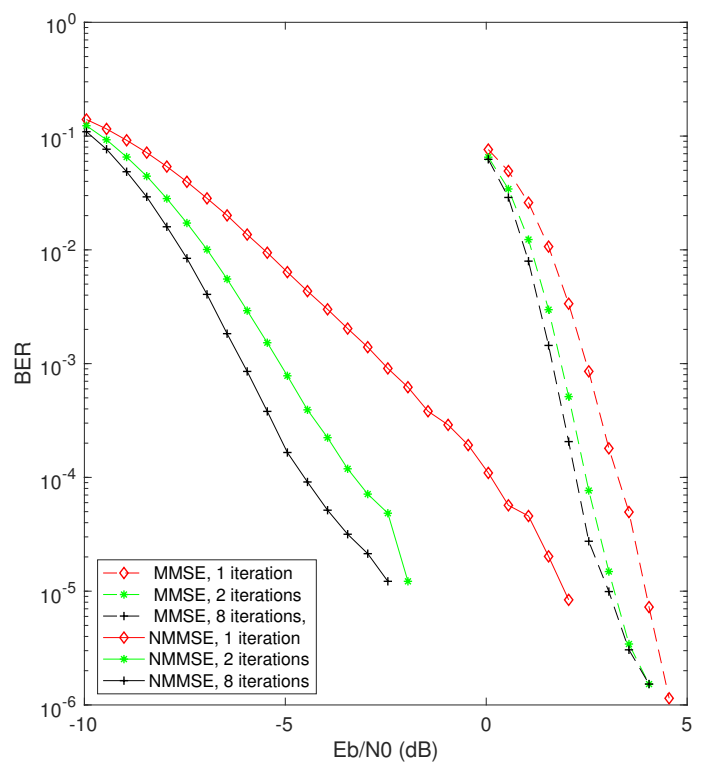

Fig. 2. Results of coded OFDM system with turbo-equalization for MMSE and nonlinear MMSE.

To compute the complexity of calculating the filter taps we consider the diagonal matrix given in (19). As described in the derivation of the equalizer, matrix $\mathbf{U}^{H} \mathbf{K}(\tilde{\mathbf{y}}) \mathbf{U}$ is a circular matrix and its entries can be calculated by passing the coefficients of diagonal entries of matrix $\mathbf{K}(\tilde{\mathbf{y}})$ through an FFT module. In this manner the complexity of calculating the equalizer taps reduces to $N \log (N)$ for a memory-less channel.

The nonlinear equalizer is implemented as in Fig. 1, and designated by NMMSE. Fig. 2 shows the result of bit error rate (BER) at the output of decoder for different number of iterations. The impulsive noise is generated for $\Gamma=0.1$ and $A=0.3$. One iteration includes one nonlinear MMSE equalization iteration followed by a turbo decoder iteration. The simulation result shows that nonlinear MMSE is effective in tackling the impulsive noise. As decoder provides more accurate data to the equalizer, our estimate of transmitted signal improves.

Fig. 3 shows the result of turbo-equalization scheme for different parameters of impulsive noise. These results are compared against MMSE equalizer for 4 iterations of turboequalization loop. For comparison, the performance of MMSE

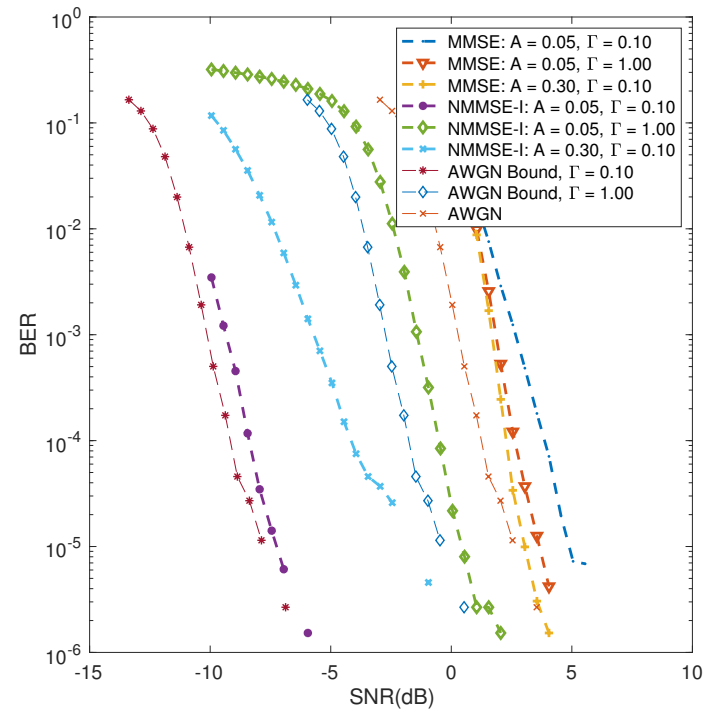

Fig. 3. Nonlinear MMSE filter used in OFDM system equipped with turboequalization for different impulsive noise parameters.

for additive white Gaussian noise (AWGN) is also shown. The MMSE performance for AWGN channel is adjusted for different $\Gamma$ 's as follows: first term, $\sigma_{0}$, is used for AWGN background noise with no impulses present. For a constant $A=0.05$ when the impulses are sparse, increasing $\Gamma$ reduces the variance of impulses which in turn improves the MMSE performance. On the other hand, as variance of background Gaussian noise (i.e., first term $\sigma_{0}$ in (4)) decreases for lower values of $\Gamma$, for instance, as $\Gamma$ changes from 1.00 to 0.10 , simulation results show that the performance of NMMSE equalizer improves with lower background noise variance. For a constant $\Gamma=0.10$, the background noise term remains intact. In this case, the lower values of $A$ the higher variances of impulses become. It can be seen from Fig. 2 the MMSE performs worse with higher variances of impulses. However, the NMMSE algorithms is performing just the opposite. The expected number of impulses are lower for $A=0.05$ relative to $A=0.30$ (see values of $a_{m}$ in (4)) which leads to a better performance.

\section{CONCLUSIONS}

Nonlinear MMSE equalizer for non-Gaussian impulsive noise was derived in this paper. The proposed nonlinear equalizer provides the needed soft values for turbo-equalization scheme. The results of simulations highlight the effectiveness of nonlinear equalizer and the impact of turbo-equalization scheme in the presence of impulsive noise. In our simulations, for the chosen code rate, the combination of nonlinear equalizer and turbo-equalization was successful in mitigating the effect of impulsive noise. For example, it was shown for sparse impulses, the performance of nonlinear MMSE equalizer is comparable to the AWGN case. Generalization of this method for a channel with ISI is the subject of our current research. 


\section{REFERENCES}

[1] K. L. Blackard, T. S. Rappaport, and C. W. Bostian, "Measurements and models of radio frequency impulsive noise for indoor wireless communications," IEEE J. Sel. Areas Commun., vol. 11, no. 7, pp. 9911001, Sep. 1993.

[2] M. Zimmermann and K. Dostert, "Analysis and modeling of impulsive noise in broad-band powerline communications," IEEE Trans Electromagn. Compat., vol. 44, no. 1, pp. 249-258, Aug. 2002.

[3] V. Degardin, M. Lienard, P. Degauque, E. Simon, and P. Laly, "Impulsive noise characterization of in-vehicle power line," IEEE Trans. Electromagn. Compat., vol. 50, no. 4, pp. 861-868, Nov. 2008.

[4] H. Dai and H. V. Poor, "Crosstalk mitigation in DMT VDSL with impulse noise," IEEE Trans. Circuits Syst. I. Fundam. Theory Appl., vol. 48, no. 10, pp. 1205-1213, Oct. 2001.

[5] X. Kuai, H. Sun, S. Zhou, and E. Cheng, "Impulsive noise mitigation in underwater acoustic ofdm systems," IEEE Trans. on Veh. Technol., vol. 65, no. 10, pp. 8190-8202, Jan. 2016.

[6] A. Bansal and A. K. Kohli, "Suppression of impulsive noise in OFDM system using imperfect channel state information," Optik-International Journal for Light and Electron Optics, vol. 127, no. 4, pp. 2111-2115, Nov. 2016.

[7] S. V. Zhidkov, "Impulsive noise suppression in OFDM-based communication systems," IEEE Trans. Consum. Electron., vol. 49, no. 4, pp. 944-948, Nov. 2003.

[8] Y. Ma, P. So, and E. Gunawan, "Performance analysis of OFDM systems for broadband power line communications under impulsive noise and multipath effects," IEEE Trans. Power Del., vol. 20, no. 2, pp. 674-682, Apr. 2005

[9] M. Mirahmadi, A. Al-Dweik, and A. Shami, "BER reduction of OFDM based broadband communication systems over multipath channels with impulsive noise," IEEE Trans. Commun., vol. 61, no. 11, pp. 4602-4615, Nov. 2013.

[10] S. V. Zhidkov, "Performance analysis and optimization of OFDM receiver with blanking nonlinearity in impulsive noise environment," IEEE Trans. Veh. Technol., vol. 55, no. 1, pp. 234-242, Jan. 2006.

[11] D.-F. Tseng, Y. S. Han, W. H. Mow, L.-C. Chang, and A. H. Vinck, "Robust clipping for OFDM transmissions over memoryless impulsive noise channels," IEEE Commun. Lett., vol. 16, no. 7, pp. 1110-1113, May 2012.

[12] K. S. Al-Mawali and Z. M. Hussain, "Adaptive-threshold clipping for impulsive noise reduction in OFDM-based power line communications," in Int. Conf. Advanced Technologies Commun., Hai Phong, Vietnam, Oct. 2009, pp. 43-48.

[13] K. Rabie and E. Alsusa, "Preprocessing-based impulsive noise reduction for power-line communications," IEEE Trans. Power Del., vol. 29, no. 4, pp. 1648-1658, Aug. 2014.

[14] C. Soltanpur, K. Rabie, B. Adebisi, and A. Wells, "Masreliez-equalized VOFDM in non-gaussian channels: Power line communication systems," IEEE Sys. J., vol. 12, no. 3, pp. 2803-2811, Jan. 2018.

[15] C. Masreliez, "Approximate non-gaussian filtering with linear state and observation relations," IEEE Trans. Autom. Control, vol. 20, no. 1, pp 107-110, Feb. 1975.

[16] X. Wang and H. Poor, "Joint channel estimation and symbol detection in Rayleigh flat-fading channels with impulsive noise," IEEE Commun. Lett., vol. 1, no. 1, pp. 19-21, Jan. 1997.

[17] D. Dardari, V. Tralli, and A. Vaccari, "A theoretical characterization of nonlinear distortion effects in ofdm systems," IEEE transactions on Communications, vol. 48, no. 10, pp. 1755-1764, Oct. 2000.

[18] D. Middleton, "Non-gaussian noise models in signal processing for telecommunications: new methods an results for class-A and class-B noise models," IEEE Trans. Inf. Theory, vol. 45, no. 4, pp. 1129-1149, May 1999.

[19] M. Tuchler, A. Singer, and R. Koetter, "Minimum mean squared error equalization using a priori information," IEEE Trans. Signal Process., vol. 50, no. 3, pp. 673-683, Mar. 2002.

[20] P. Amirshahi, S. Navidpour, and M. Kavehrad, "Performance analysis of uncoded and coded OFDM broadband transmission over low voltage power-line channels with impulsive noise," IEEE Trans. Power Del., vol. 21, no. 4, pp. 1927-1934, Oct. 2006.

[21] D. Middleton, "Canonical and quasi-canonical probability models of class A interference," IEEE Trans. Electromagn. Compat., vol. EMC25, no. 2, pp. 76-106, May 1983.

[22] B. Adebisi, Broadband Transmission over Indoor Power-Line channels. Ph.D. dissertation, School of Computing and Communications: Lancaster University, UK, 2009.
[23] K. Rabie and E. Alsusa, "Effective noise cancellation using single-carrier FDMA transmission in power-line channels," IEEE Trans. Power Del., vol. 29, no. 5, pp. 2110-2117, Oct. 2014.

[24] T. Shongwe, A. Han Vinck, and H. C. Ferreira, "A study on impulse noise and its models," SAIEE Africa Research J., vol. 106, no. 3, pp. 119-131, 2015.

[25] R. B. Ash, B. Robert, and C. A. Doleans-Dade, Probability and measure theory. New York: Academic Press, 2000.

[26] R. Koetter, S. A.C., and T. M., "Turbo equalization," IEEE Signal Process. Mag., vol. 21, no. 1, pp. 67-80, Feb. 2004.

[27] S. Zhidkov, "Analysis and comparison of several simple impulsive noise mitigation schemes for ofdm receivers," IEEE Trans. Commun., vol. 56, no. 1, pp. 5-9, Jan. 2008.

[28] K. Fang, L. Rugini, and G. Leus, "Low-complexity block turbo equalization for ofdm systems in time-varying channels," IEEE Trans. on Signal Process., vol. 56, no. 11, pp. 5555-5566, Aug. 2008.

[29] M. Tuchler, R. Koetter, and A. Singer, "Turbo equalization: principles and new results," IEEE Trans. on Commun., vol. 50, no. 5, pp. 754-767, May 2002.

[30] X. Wang and H. Poor, "Iterative (turbo) soft interference cancellation and decoding for coded CDMA," IEEE Trans. Commun., vol. 47, no. 1 p. 10461061, July 1999.

[31] E. L. Lehmann and G. Casella, Theory of Point Estimation (2nd ed.). New York, NY:Springer-Verlag, 1998.

[32] D. Raphaeli and Y. Zarai, "Combined turbo equalization and turbo decoding," IEEE Commun. Lett., vol. 2, no. 4, pp. pp.107-109, Apr. 1998.

[33] S. Lin and J. J. Costello, Error Control Coding. Englewood Cliffs, NJ: Prentice Hall, 1983. 